\title{
A!
}

This is an electronic reprint of the original article.

This reprint may differ from the original in pagination and typographic detail.

Puska, M. J.; Nieminen, R. M.; Manninen, Matti; Chakraborty, Bulbul; Holloway, S.; Nørskov, J. K.

\section{Quantum motion of chemisorbed hydrogen on Ni surfaces}

\section{Published in:}

Physical Review Letters

DOI:

10.1103/PhysRevLett.51.1081

Published: 19/09/1983

\section{Document Version}

Publisher's PDF, also known as Version of record

Please cite the original version:

Puska, M. J., Nieminen, R. M., Manninen, M., Chakraborty, B., Holloway, S., \& Nørskov, J. K. (1983). Quantum motion of chemisorbed hydrogen on Ni surfaces. Physical Review Letters, 51(12), 1081-1084.

https://doi.org/10.1103/PhysRevLett.51.1081

This material is protected by copyright and other intellectual property rights, and duplication or sale of all or part of any of the repository collections is not permitted, except that material may be duplicated by you for your research use or educational purposes in electronic or print form. You must obtain permission for any other use. Electronic or print copies may not be offered, whether for sale or otherwise to anyone who is not an authorised user. 


\title{
Quantum Motion of Chemisorbed Hydrogen on Ni Surfaces
}

\author{
M. J. Puska \\ Laboratory of Physics, Helsinki University of Technology, 02150 Espoo, Finland \\ and \\ R. M. Nieminen \\ Department of Physics, University of Jyväskylä, 40150 Jyväskylä, Finland \\ and \\ M. Manninen \\ Research Institute for Theoretical Physics, University of Helsinki, 00170 Helsinki 17, Finland \\ and \\ Bulbul Chakraborty, (a) S. Holloway, and J. K. Nфrskov \\ NORDITA, 2100 Copenhagen $\emptyset$, Denmark \\ (Received 30 June 1983)

\begin{abstract}
Quantum mechanical energy levels and wave functions have been calculated for the motion of chemisorbed hydrogen atoms on Ni surfaces. The results show considerable quantum effects for the adatom in both the ground and the excited states. The description of the adparticles as being delocalized along the surface offers a novel interpretation of several phenomena, in particular the vibrational excitations.
\end{abstract} \\ PACS numbers: $71.45 . \mathrm{Nt}, 68.30 .+\mathrm{z}$
}

The nature of chemisorbed hydrogen on metal surfaces has been a subject of considerable interest. Usually the hydrogen atoms are considered chemisorbed at a well-defined site on the surface, and the elementary excitations are small vibrations around the equilibrium position. In the present Letter we advocate a completely different view. We demonstrate that the motion of chemisorbed hydrogen exhibits pronounced quantum effects. The hydrogen atom is to a large extent delocalized in both the ground-state and the excitedstate configurations. A proper description of the properties of these systems can only be given in terms of hydrogen energy bands. A similar picture has previously been proposed to explain qualitatively the structural disorder observed in lowenergy electron-diffraction (LEED) experiments for hydrogen on $\mathrm{Ni}(111){ }^{1}$ This new description of chemisorbed hydrogen can explain a number of experimental observations including the vibrational excitation spectrum as measured for example by electron-energy-loss spectroscopy (EELS).

The starting point for the description of the chemisorbed hydrogen is a calculation of the full adiabatic potential-energy surface for hydrogen outside the surface in question. This involves the calculation of the hydrogen-surface interaction energy for all possible hydrogen positions. Even within the local density approximation to the den- sity functional theory, ${ }^{2}$ such a calculation is incredibly cumbersome without further approximations. The effective-medium theory ${ }^{3}$ is well suited for this purpose. The details of the approach have been described by N $\not$ rskov and coworkers, ${ }^{4}$ who have also shown it to give good chemisorption energies and bond lengths when compared to both other theoretical approaches and experiment. The calculated potential for hydrogen outside a $\mathrm{Ni}(100)$ surface is shown in Figs. $1(a)$ and 1(b). Contours of constant potential energy are shown for two cuts, perpendicular and parallel to the surface. It is seen that the potential is very anharmonic, resulting in a strong coupling between motion parallel and perpendicular to the surface. Clearly a full determination of the vibrational excitation spectrum within the harmonic approximation is not possible.

For a proper description of the ground state and the excitation spectrum, the three-dimensional Schrödinger equation must be solved for the hydrogen atom within the calculated potential. In the present work, this is accomplished by a discrete-mesh numerical relaxation technique, the exact details of which will be published elsewhere. ${ }^{5}$

The potentials used have been calculated for a single hydrogen adatom on a semi-infinite surface and no attempt has been made to include correla- 
tion effects due to finite hydrogen concentrations. We shall return to this question later. The coupling of the chemisorbed hydrogen to lattice distortions is found to be very weak and self-trapping

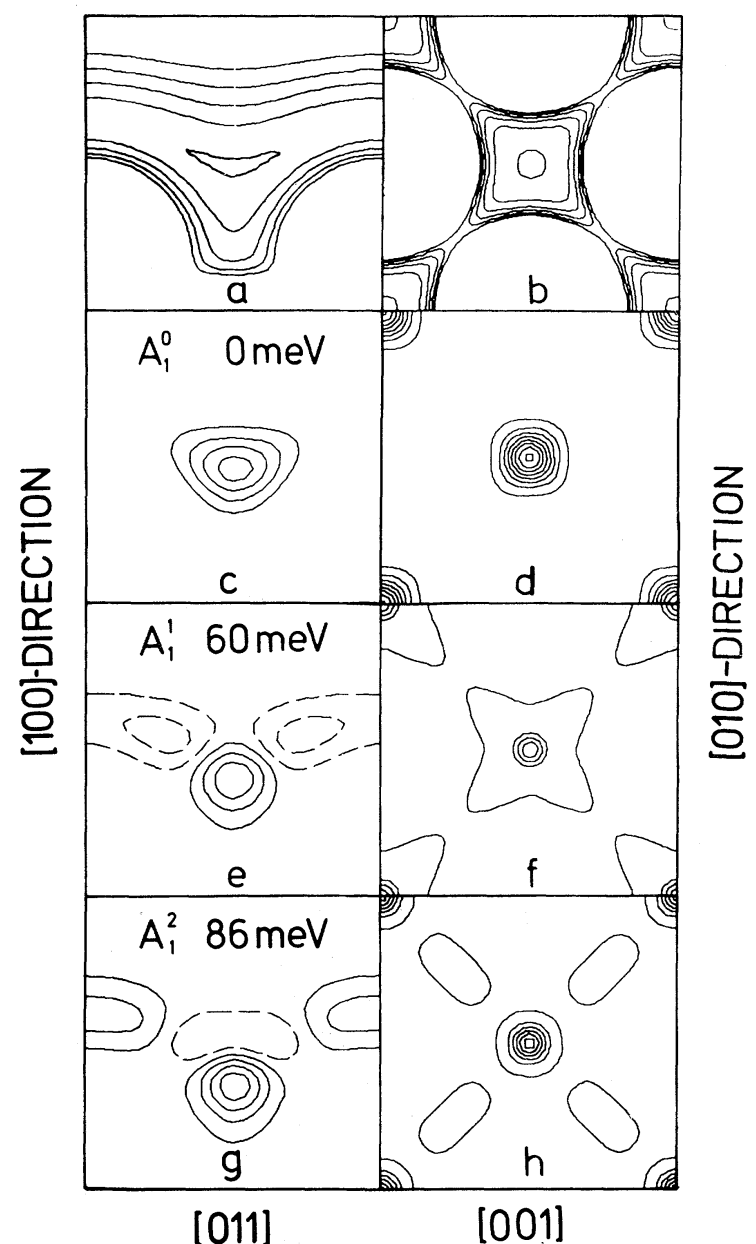

FIG. 1. Potential, $A_{1}$ wave functions, and densities for hydrogen chemisorbed on the Ni(100) surface. The left panel shows the potential and wave functions in a vertical plane along the $\langle 110\rangle$ direction through the fourfold center position where the potential has its minimum. The lengths of the cuts are the Ni nearestneighbor distance $\left(4.7 a_{0}\right)$ both in the parallel and the perpendicular directions. At the top of the right panel the potential is shown in a cut parallel to the surface through the absolute minimum. Underneath, we show the hydrogen densities, integrated perpendicular to the surface in the same parallel cut. In the right panel the cuts are one Ni lattice constant $\left(6.65 a_{0}\right)$ in each direction. The lowest energy contour shown is -2.70 $\mathrm{eV}$ and the spacing between contours is 0.15 and 0.22 $\mathrm{eV}$ for (a) and (b), respectively. The wave-function contours are shown with a constant spacing, and with the same minimum contour value in all cuts. The same is true for the density contours. Dashed lines denote negative contours. All wave functions are evaluated at $\overline{\boldsymbol{\Gamma}}$. effects have therefore been neglected.

Turning now to the results, we show in Fig. 2 the energy bands for hydrogen on $\mathrm{Ni}(100)$ along the high-symmetry directions in reciprocal space. For simplicity, we first concentrate on the $A_{1}$ states, belonging to the totally symmetric representation of the surface point group $\left(C_{4 v}\right.$ in this case).

The lowest band in Fig. 2 is essentially dispersionless, implying that, in its ground state, the chemisorbed hydrogen is fairly well localized within the unit cell on the $\mathrm{Ni}(100)$ surface. This is also illustrated by the wave function and hydrogen density contours shown in Figs. 1(c) and 1(d). The first excited band has a $5-\mathrm{meV}$ dispersion and a correspondingly large wave-function overlap between adjacent unit cells, as seen from Figs. 1(e) and 1(f). The states around the bottom of the band have "bonding" characteristics between adjacent unit cells [see Figs. 1(c), 1(b), and $1(\mathrm{~g})$ ], whereas those around the top are "antibonding." Figure 1(e) shows very clearly that the first excited band corresponds to "parallel" motion along the surface, whereby the wave function closely follows the potential energy valley, indicated in Fig. 1(a). As a consequence of the corrugation in the potential, this leads to a substantial variation of the wave function normal to the

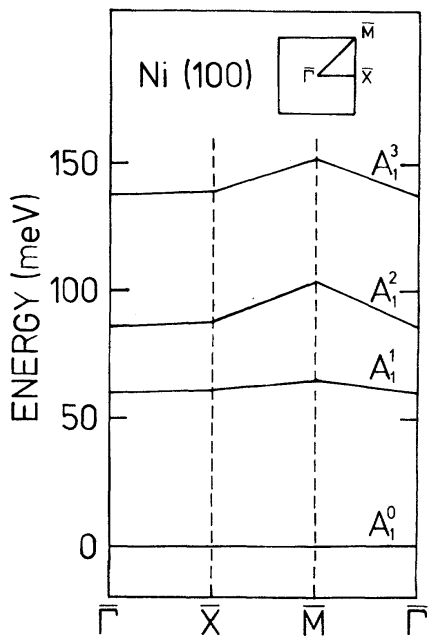

FIG. 2. The band structure for hydrogen chemisorbed on the Ni(100) surface shown along the high-symmetry directions indicated in the inset. Only the states belonging to the $A_{1}$ representation of the $C_{4 v}$ point group are shown. The zero of energy is the ground-state energy $(-2.6 \mathrm{eV})$ at the $\Gamma$ point. This includes a zeropoint energy of $0.1 \mathrm{eV}$. In the inset the Brillouin zone has been rotated $45^{\circ}$ relative to the convention used in Fig. 1. 
surface. The states in the second excited band exhibit more dispersion than the first. It is also evident from Fig. $1(\mathrm{~g})$ that the wave function has picked up an appreciable degree of "perpendicularly excited" character. If one makes a harmonic analysis of the hydrogen motion perpendicular to the surface above the center site, the energy of the first excited state is $76 \mathrm{meV}^{4}$ it is interesting to note that the energy range of the second band in Fig. 2 is close to this energy. The higher excited bands correspond to even more complicated wave functions, reflecting the nonseparable nature of the potential.

In Table I we summarize the results for the $A_{1}$ and $E$ bands for both the $\mathrm{Ni}(100)$ and (111) surfaces. The main difference between the two surfaces is that the bandwidths are much la rger for $\mathrm{Ni}(111)$. This is not because the potential energy barriers are lower on the $\mathrm{Ni}(111)$ surface, but because the distance between the minima, in the triangular sites of the (111) surface, is smaller. This increases the overlap between the hydrogen wave functions centered at each minimum. As for the (100) surface, the first excited band corresponds to parallel motion along the surface whereas the second excited state has a pronounced perpendicular component. The second excited band is again close in energy to the perpendicular vibrational frequency in harmonic analysis. ${ }^{4}$

In Table I the vibrational excitation energies measured by EELS under specular reflection conditions are shown for both the $\mathrm{Ni}(100)$ and (111) surfaces. In the present picture, these excitations correspond to vertical $\left(\Delta k_{\|}=0\right)$ transitions between the ground state and excited $A_{1}$-symmetry bands. For nonspecular conditions, nonvertical and $A_{1} \rightarrow E$ transitions a re also symmetry allowed. It is seen that there is rather good agreement between the experimental and theoretical excitation energies, taking into account the approximations involved in the potential construction. In particular, we can explain the existence of two loss peaks for the $\mathrm{Ni}(111)$ surface. On the $\mathrm{Ni}(100)$ surface only one clear peak is observed experimentally. ${ }^{6}$ The excitations to the second (perpendicular) band are most intensive for the $\mathrm{Ni}(111)$ surface ${ }^{7}$ and by analogy we interpret the $\mathrm{Ni}(100)$ loss peak to be due to excitations to the second band. In this connection it is interesting to note that there is a weak structure in the EELS spectrum for $\mathrm{Ni}(100)$ at lower energies, ${ }^{6}$ which could be due to the first excited band. For hydrogen on the $\mathrm{Ni}(111)$ surface the EELS spectra have also been recorded for off-specular scattering
TABLE I. Calculated centers and widths for the lowest $A_{1}$ and $E$ symmetry bands compared with the experimentally observed EELS energies for hydrogen chemisorbed on the $\mathrm{Ni}(100)$ and (111) surfaces. The band center is defined as the average of the top and the bottom of the band. Note that the band center is not necessarily equal to the center of gravity of the band (see, e.g., Fig. 2). Comparisons between theory and experiment are therefore only valid to within half the bandwidth.

\begin{tabular}{lrccrcc}
\hline \hline & $\begin{array}{c}\text { Band } \\
\text { center } \\
(\mathrm{meV})\end{array}$ & $\begin{array}{c}\text { Ni(100) } \\
\text { Band- } \\
\text { width } \\
(\mathrm{meV})\end{array}$ & $\begin{array}{c}\text { Experi- } \\
\text { ment }^{\mathrm{a}} \\
(\mathrm{meV})\end{array}$ & $\begin{array}{c}\text { Band } \\
\text { center } \\
(\mathrm{meV})\end{array}$ & $\begin{array}{c}\text { Ni(111) } \\
\text { Band- } \\
\text { width } \\
(\mathrm{meV})\end{array}$ & $\begin{array}{c}\text { Experi- } \\
\text { ment }^{\mathrm{b}} \\
(\mathrm{meV})\end{array}$ \\
\hline$A_{1}{ }^{0}$ & 0 & $<1$ & & 2 & 4 & \\
$A_{1}{ }^{1}$ & 62 & 5 & & 74 & 43 & 88 \\
$A_{1}{ }^{2}$ & 95 & 18 & 74 & 138 & 40 & 139 \\
$A_{1}{ }^{3}$ & 145 & 14 & & & & \\
$E^{1}$ & 45 & $<1$ & & 40 & 26 & \\
$E^{2}$ & 80 & 14 & & 136 & 58 & \\
\hline \hline
\end{tabular}

${ }^{\mathrm{a}}$ Ref. 6.

${ }^{\mathrm{b}}$ Ref. 7 .

conditions. $^{7}$ As seen in Table I the lowest $E$ state is so low in energy that it would lie in the background in the EELS spectrum. The second excited $E$ band lies exactly on top of the second excited $A_{1}$ band. This could explain why no new peaks are observed experimentally under offspecular conditions. ${ }^{7}$

We have also calculated the energy spectrum for deuterium. The ratio of the hydrogen and deuterium excitation energies are always close to $\sqrt{2}$, as is also observed experimentally. ${ }^{6,7}$

Since the ground-state bands a re flat compared to the relevant temperature in the EELS experiments, all states within the ground-state band are populated, and vertical transitions at any point in the Brillouin zone should be observable. Consequently the experimental loss peaks should have the width of the corresponding excited band, in the limit of zero hydrogen coverage. The observed loss at $74 \mathrm{meV}$ for the $\mathrm{Ni}(100)$ surface is slightly narrower ${ }^{6}$ than the calculated bandwidth. For the (111) surfaces the observed peaks are also narrower than the calculated bands, but the trend that the (111) bands are much broader than the (100) bands is clearly observed. ${ }^{7}$ It is to be expected that the hydrogen bandwidth is smaller at the finite coverages where the experiments are performed. At high coverage, blocking by neighboring hydrogen atoms will restrict the mobility and thereby decrease the bandwidth. Typi- 
cally the overlayer structures considered experimentally are rather open, with at least nearestneighbor sites free from the other hydrogen atoms. We therefore expect the qualitative picture developed above to be still applicable. The recent observation for hydrogen on $\operatorname{Pd}(100),{ }^{8}$ of a decrease in the width of the EELS loss peak with increasing coverage, could be explained by the effect described above.

In summary, we have calculated the hydrogen band structure on the $\mathrm{Ni}(100)$ and (111) surfaces. The hydrogen bands, in particular the excited states, have a considerable width, suggesting a high mobility on the surface. The calculated energy spectra can explain the observed vibrational losses and in particular the occurrence of two vibrational peaks on the (111) surface, corresponding to a perpendicular and a parallel mode. There are also a number of other experimental observations for hydrogen chemisorption systems which are most easily understood with a delocalized picture. LEED observations show a large degree of disorder on the $\mathrm{Ni}(111)$ surface as mentioned above, ${ }^{1}$ and simila $r$ observations exist for $\mathrm{Ni}(100){ }^{6}$ The hydrogen mobility on these surfaces is extremely high even at low temperatures. ${ }^{1}$ Also, to explain the observed kinetics of some surface reactions involving hydrogen, it has been necessary to involve an unusually large surface mobility for hydrogen. ${ }^{9}$ This was taken as evidence for nonthermalized (hot) adsorbate atoms moving fast on the surface. If the hydrogen atoms are as delocalized as described in the present paper, the mobility would be high even at room temperature and it would be unnecessary to invoke the concept of hot adsorbates in this context.

Similar delocalization phenomena as those considered here for hydrogen on Ni surfaces are also expected for other metal surfaces, where the effective-medium theory predicts qualitatively similar potentials. ${ }^{4}$

Discussions with P. Jena are gratefully acknowledged.

\footnotetext{
(a) Present address: Indian Institute of Science, Bangalore-560012, India.

${ }^{1}$ K. Christmann, R. J. Behm, G. Ertl, M. A. van
} Hove, and W. H. Weinberg, J. Chem. Phys. ㅁ, 4168 (1979).

${ }^{2}$ W. Kohn and L. J. Sham, Phys. Rev. 140, A1133 (1965); O. Gunnarson and B. I. Lundqvist, Phys. Rev. B 13, 4274 (1976).

$\overline{3}$ J. K. Nørskov and N. D. Lang, Phys. Rev. B 21, 2136 (1980); M. J. Stott and E. Zaremba, Phys. Rev. B 22, 1564 (1980).

JJ. K. Nбrskov, Phys. Rev. B 26, 2875 (1982), and Phys. Rev. Lett. 48,1620 (1982); P. Nordlander, S. Holloway, and J. K. Nørskov, to be published.

${ }^{5}$ M. J. Puska and R. M. Nieminen, to be published.

${ }^{6} \mathrm{~S}$. Andersson, Chem. Phys. Lett. 55, 185 (1978).

${ }^{7}$ W. Ho, N. J. DiNardo, and E. W. Plummer, J. Vac. Sci. Tech. 17, 134 (1980).

${ }^{8}$ C. Nyberg and C. G. Tenstål, Solid State Commun. 44, 251 (1982).

${ }^{9} \mathrm{~J}$. Harris, B. Kasemo, and E. Törnqvist, Surf. Sci. 105, L288 (1981). 